\title{
Dynamic Real Time Distortion Management Over Multimedia Downlink CDMA
}

\author{
Andres Kwasinski, Zhu Han and K.J. Ray Liu \\ Department of Electrical and Computer Engineering, University of Maryland, College Park, Maryland, USA. \\ $\{$ ak,hanzhu,kjrliu\}@umd.edu
}

\begin{abstract}
In CDMA networks it is possible to increase the maximum number of users of real time applications by smoothly increasing each end-to-end distortion. In this paper we study a protocol for downlink CDMA that allocates source coding rate, channel coding rate and transmitted power based on each user's channel state. The allocation of resources is performed with the constraints of users' maximum distortion and base station's maximum transmitted power in such a way that users operate under carefully controlled distortions. The optimization goal is to minimize the overall distortion in downlink singlecell systems. When the system is highly loaded, the idea for the proposed solution is to initialize the resource allocation with that corresponding to maximum distortion for all users, and then incrementally allocate the remaining transmitted power quota to the user that, at each step, can reduce the distortion with the least use of power. We show that this user must have a high distortion, experience a good channel condition, or generate small interference. We also extend our study to include speech's talk spurt-silence nature and to consider a dynamic model with different offered loads. From simulation results, the proposed algorithm fundamentally reduces the distortions and the necessary maximum transmitted power when the number of users is large, compared with a traditional CDMA scheme (with no distortion control).
\end{abstract}

\section{INTRODUCTION}

Code Division Multiple Access (CDMA) is characterized by all users transmitting simultaneously over the same frequency band. Ideally, users do not interfere with each other because each user is assigned a different spread spectrum code. Because perfect separation between codes is not achievable in practice, inter-user interference limits the capacity and the maximal number of users that can be simultaneously supported. Resource allocation such as rate adaptation and power control is an important means to combat this interference, increase the number of admitted users, and maintain the end-to-end quality during a call. By carefully choosing the allocation of source and channel coding rates it is possible to control the source encoder's output quality and level of channel errors protection. Also, power control is a technique that allows maintaining the received signal-to-interferenceplus-noise-ratio (SINR) at a desire level. Consequently, the quality of the received signals can be carefully controlled according to channel conditions. In this context the problem is how to increase the system performance, by cleverly allocating these resources while also taking into consideration practical constraints.

Distortion-based and CDMA resource allocation are intensively researched areas of study. In [1], the authors developed a source encoding assisted multiple access protocol to increase a TDMA system capacity during congestions by selectively adapting the source encoding rate. In [2], the authors formulated a CDMA resource allocation problem for different Quality of Service (QoS) requirements. An adapted power and joint source channel coding video transmission scheme over multi-access networks that minimizes power consumption was presented in [3]. Although, the problem solved in this work is different, the proposed solution in this paper is partially inspired in [3]. One area of research considers power allocation based on defined utilities. Such is the case in [4], [5], where the authors used a dynamic pricing scheme with cooperation between the mobiles and the base stations to maximize the total system utility. In this paper we focus on real-time sources, thus we consider end-to-end distortion. In [6], the authors formulated the resource allocation problem as a constrained optimization one and used approximations to have a simple solution form. In terms of methodology, we observed that the solutions proposed in existing works could be classified into two kinds. The first kind of methods uses convex or linear approximations and applies Lagrangian or convex optimization methods. The problem here is that there exists a mismatch between real situations and the approximations. The second kind of methods applies nonlinear programming. However the complexity grows fast as the number of users increases. Therefore, the goal is to find a fast algorithm with relatively good performances. In [7] we presented the idea of allocating resources in the downlink of a CDMA system through a controlled degradation of quality as the network becomes increasingly loaded.

In this paper, we develop and present a fast resource allocation algorithm for distortion management in the downlink of a single-cell CDMA systems, based on the idea introduced in [7]. The goal is to allocate resources to all users while reducing overall system distortion. Therefore, the design is constrained by the maximum transmitted power from the base station and the maximum distortion for each user. If the network is lightly loaded, the allocation algorithm assigns resources in such a way that all users operates at minimal distortion. When the network becomes highly loaded it would not be possible to make all users operate with minimal distortion even when using the maximum available transmit power. In this situation, the algorithm first assigns to each user those resources that correspond to maximum distortion. If there is transmitted power left, the algorithm assigns some extra power to the user who will use up less power while reducing more distortion. To deserve an assignment that reduces distortion, a user must have a reduced rate (high distortion), good channel condition, or generate small interference to others. After every 
assignment, the algorithm evaluates whether the transmitted power has been used up. If not, it will continue assigning power to reduce distortion.

The organization of this paper is as follows: In Section II, we explain the system model and describe the proposed cross-layer protocol. In Section III, we formulate the problem and develop the allocation algorithm for downlink distortion management. In Section IV, we present simulation studies. Section V presents the conclusions.

\section{System Model AND Protocol Description}

Consider the downlink of a single cell CDMA system with $\mathrm{N}$ users. We assume the system is synchronous and each user is assigned a unique spreading code within each cell. Because of the multipath environment, the orthogonality between codes could not be guaranteed and each mobile user is subject to interferences from other users in the cell [9]. The SINR of mobile $i$ is given by:

$$
\Gamma_{i}=\frac{W}{R} \frac{P_{i} G_{i}}{G_{i} \sum_{\substack{k=1 \\ k \neq i}}^{N} \alpha_{k i} P_{k}+\sigma^{2}}
$$

where $W$ is the total bandwidth, $R$ is the transmit rate, $P_{i}$ is the transmitted power from the base station for mobile $i, \alpha_{k i}$ is orthogonality factor between mobile $k$ and mobile $i, G_{i}$ is the corresponding path loss, and mobiles' thermal noise levels are assumed to be the same as $\sigma^{2} . W / R$ is the processing gain. $\alpha_{k i}$ is the orthogonality factor which represents the fraction of the received downlink power that is converted by multipath into the intra-cell interference. The higher the value, the more the orthogonality loss. We assume the fading profiles are the same and $\alpha=\alpha_{k i}, \forall i, k$. In [10], for independent Rayleigh fading $\theta_{i}^{l}$, the average orthogonality factor is approximated by:

$$
\alpha=0.81-\frac{0.81 \sum_{l=1}^{L}\left(E\left(\left|\theta_{i}^{l}\right|^{2}\right)\right)^{2}}{\left(\sum_{l=1}^{L} E\left(\left|\theta_{i}^{l}\right|^{2}\right)\right)^{2}} .
$$

Fig. 1 shows the block diagram of the proposed cross layer protocol to manage interference by controlling the users' source rates, channel coding rates and transmitted powers. The protocol is located in the base station and allocates rates and powers to all the users based on the channel conditions.

In the proposed system, the real time source encoder has the key property that the output rate can be externally controlled. This can be implemented using either variable rate or embedded encoders. In the first case, the encoder generates one bit stream for each of the possible coding rates. Only one of these will be selected and transmitted based on the rate assignment. Using embedded encoders presents the disadvantage of typically lower coding efficiency and the advantage that only one bit stream is generated, making the adaptation to the rate assignment simply by dropping as many bits as necessary from the end of the bit stream. Although the "bit dropping mechanism" is exclusive to the embedded stream, we will loosely use this term to represent a reduction in the source rate,

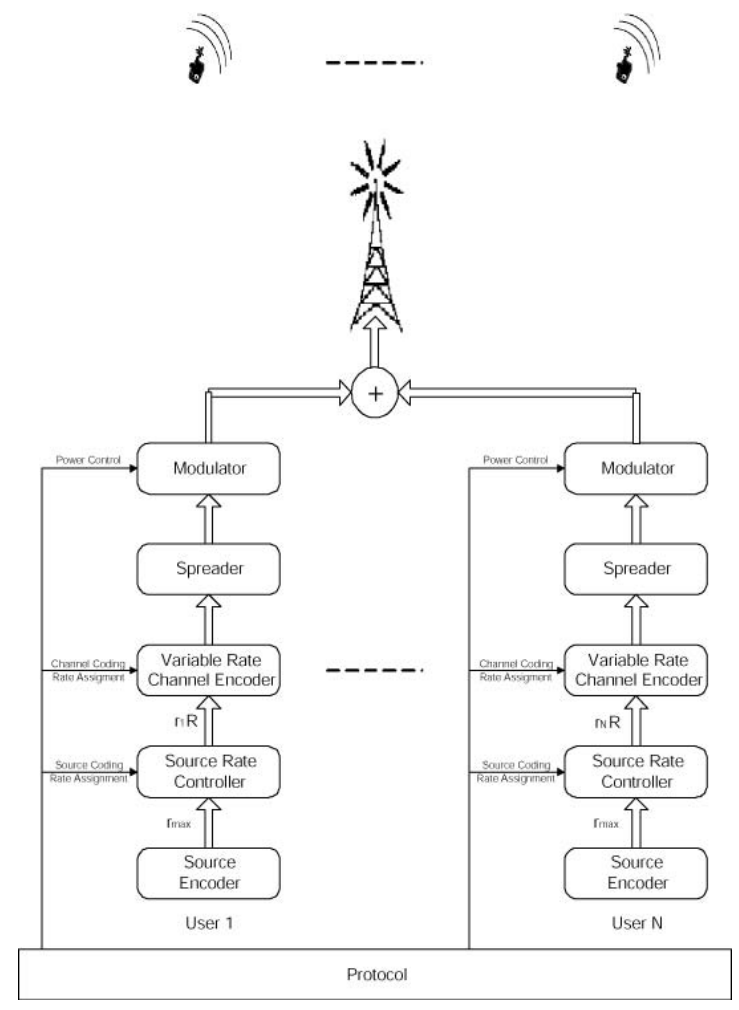

Fig. 1. Protocol Block Diagram

regardless of the particular source encoder implementation. We assume the source coder have the maximal output rate $r_{\max }$ bits/s and the source rate controller have the output rate $r_{i} R$ bits/s, where $r_{i}$ is the variable channel coding rate. We use BPSK modulation with power control in the modulator. Also, for simplicity, we assume that all the transmitted bits are equally important for error protection purposes.

We have observed during simulations that, for the same level of distortion, channel-induced errors are perceptibly more annoying than source encoding distortion. Because if this reason, a design goal, called hereafter quality goal, would be that channel induced errors would account for a small percentage of the overall end-to-end distortion. Thus, the design will be constrained by the condition of meeting a target SINR that achieves the desired small channel-induced distortion. Note that the target SINR required to satisfy the quality goal is a function of the source rate. This is because reducing source rate corresponds to increasing the source frame error protection, which, in turn, lowers the SINR needed to achieve the same quality goal. Thus, by reducing some or all calls' source rate, it is possible to lower transmit power and interference at the cost of higher source encoding distortion but without increasing channel induced errors. Furthermore, we have found through simulations using different configurations of Rate Compatible Punctured Convolutional (RCPC) codes [8] that the SINR as a function of channel coding rate, when transmit rate is fixed, can be approximated accurately by

$$
\gamma_{i}=2^{A r_{i}+B},
$$


where $\gamma_{i}$ is the target SINR required to achieved the desired quality goal $\left(\Gamma_{i} \geq \gamma_{i}, \forall i\right), A$ and $B$ are the parameters of the error control coding scheme, and $r_{i}$ is the channel coding rate.

Define $f_{i}\left(r_{i} R\right)$ as the distortion-rate function of the $i^{\text {th }}$ user's source coder encoding at rate $r_{i} R$. In most well designed encoders, $f_{i}$ is a convex and decreasing function. The minimum distortion occurs at maximum source rate $r_{\max }$. Furthermore, we assume that the source encoder distortionrate function [11], [12] is:

$$
f_{i}=\delta 2^{2 k\left(r_{\max }-r_{i} R\right)}
$$

where $\delta$ is the minimum distortion and $k$ is a parameter depending on the encoder. This is a very general form that applies to both the cases of uncorrelated Gaussian source with squared-error distortion and when the high-rate approximation holds. In practice, the distortion-rate performance of real complex encoders does not strictly follow this rule for all encoding rates. Nevertheless, we noticed that by determining the parameter $k$ from simulations, this function is a good representation of a tight upper bound for the real distortionrate characteristic and can be safely used as the representation for the worst-case behavior of the encoder. Even more, note that the approximation (4) can effectively represent the end-toend behavior because the quality goal keeps the contribution of channel induced errors to the overall distortion within negligible values. Define $D=2^{2 k r_{\max }} / \delta$, the normalized distortion is given by:

$$
D_{i}\left(r_{i} R\right)=\frac{f_{i}}{\delta}=D 2^{-2 k r_{i} R} .
$$

\section{Real Time Distortion Management}

\section{A. Problem Formulation}

As the system admits more users, the increase in interference will prevent allocating resources so that all users have the minimum distortion, even when transmitting using the maximum power. Therefore, some users will have to operate at an increased distortion. Thus, the problem is to decide which user will be affected and how these users will increase their distortions.

The basic effect of the adaptation that solves the allocation problem is to choose for each user a target SINR. From the discussion in the previous section, we can see that each target SINR is associated to a distinct source encoding rate, or equivalently, a channel coding rate $r_{i}$. Therefore, the adaptation goal would be to find the channel coding rate for each user that minimize the overall system distortion, under the constraint that each user's distortion is smaller than a maximum acceptable value and that the overall transmitted power from the base station, $P_{\text {sum }}=\sum_{i=1}^{N} P_{i}$, is bounded. The problem is formulated as:

$$
\min _{r_{i}} \sum_{i=1}^{N} D_{i}
$$

subject to $\begin{cases}\text { Distortion Range: } & 1 \leq D_{i} \leq D_{\max }, \forall i, \\ \text { Transmitted Power: } & P_{\text {sum }} \leq P_{\max }, \\ \text { Meet quality goal. } & \end{cases}$

where $P_{\max }$ is the maximum transmit power available at the base station and $D_{\max }$ is the maximal acceptable distortion. In this paper, for simplicity, we assume all users have the same $D_{\max }$. Note that $r_{i}$ is implicitly constrained by the combination of equation (5) with the above distortion range constraint.

The problem in (6) is a nonlinear nonconvex problem and there might be many local minima. It is very difficult to solve by Lagrangian method or nonlinear integer programming. Moreover, the computation complexity will grow exponential as the number of users increases. In order to fit a real CDMA system with large number of users, we need to develop a fast algorithm with relatively good performances.

\section{B. Resource Allocation Algorithm}

As noted, when the system is lightly crowded, each user could have the minimum distortion and the necessary total transmitted power could still be less than the maximum transmitted power available from the base station. As the system becomes more crowded the system might not be able to let every user operate at minimum distortion. In this operational condition, there is a need to have a graceful distortion control so that those users in bad channel condition or who introduce too much interference to others may sacrifice their performance slightly by increasing their distortion in a controlled way. Note that the constraint on the channel induced errors (the quality goal) will allow the increase in distortion to be smooth, controllable, and predictable. This is because the dominant process in increasing distortion is the reduction in source encoding rate, thus the system behavior follows the rate-distortion curve. This will keep the random and subjectively more annoying channel-induced distortion at a negligible value. Channel induced distortion is kept at a sufficiently small value by appropriately setting the rates and powers. By doing this, the system will use the limited power to reduce co-channel interferences, minimize the overall system distortion, and increase the total number of users.

In order to decide who will be operating at a lower distortion we consider the effect that a change in each user's distortion have on the total transmit power. First we need to have a simple approximation for $P_{\text {sum. }}$. Define

$$
T_{i}=\frac{2^{A r_{i}+B} R}{W}=\frac{P_{i} G_{i}}{G_{i} \sum_{k \neq i} \alpha_{k i} P_{k}+\sigma^{2}} .
$$

If the processing gain is large, i.e., $W / R$ is large, $T_{i}$ is small. We know that $\alpha_{k i}<1$, thus $\alpha_{k i} T_{i}$ is also small. Therefore, we have $P_{\text {sum }}=\mathbf{1}^{T}[\mathbf{I}-\mathbf{F}]^{-1} \mathbf{u} \approx \mathbf{1}^{T}[\mathbf{I}+\mathbf{F}] \mathbf{u}$, where $\mathbf{1}=[1 \ldots 1]^{T}$, $\mathbf{u}=\left[u_{1}, \ldots u_{N}\right]^{T}$ with $u_{i}=\sigma^{2} T_{i} / G_{i}$, and

$$
[\mathbf{F}]_{j i}= \begin{cases}0 & \text { if } j=i, \\ \alpha_{j i} T_{i} & \text { if } j \neq i .\end{cases}
$$


Therefore,

$$
P_{\text {sum }} \approx \sum_{i=1}^{N} \frac{\sigma^{2} T_{i}}{G_{i}}+\sum_{i=1}^{N} \sum_{j \neq i}^{N} \frac{\sigma^{2} \alpha_{j i} T_{i} T_{j}}{G_{j}},
$$

We find the gradient of the overall transmitted power with respect to each user's distortion, $g_{i}$. The gradient can be written as a function of three differentials, as follows:

$$
g_{i}=\frac{\partial P_{\text {sum }}}{\partial D_{i}}=\frac{\partial P_{\text {sum }}}{\partial T_{i}} \frac{\partial T_{i}}{\partial r_{i}} / \frac{\partial D_{i}}{\partial r_{i}},
$$

where

$$
\begin{aligned}
\frac{\partial P_{\text {sum }}}{\partial T_{i}} & =\frac{\sigma^{2}}{G_{i}}+\sum_{j \neq i}^{N} \frac{\sigma^{2} \alpha_{j i} T_{j}}{G_{j}}, \\
\frac{\partial T_{i}}{r_{i}} & =\frac{A R 2^{A r_{i}+B} \ln 2}{W}, \\
\frac{\partial D_{i}}{\partial r_{i}} & =-2 k D R 2^{-2 k r_{i} R} \ln 2 .
\end{aligned}
$$

Therefore, the final gradient can be written as:

$$
g_{i}=C 2^{(A+2 k R) r_{i}}\left(\frac{1}{G_{i}}+\sum_{j \neq i}^{N} \frac{\alpha_{j i} T_{j}}{G_{j}}\right)
$$

where $C$ is a negative constant. The absolute value of $g_{i}$ is determined by three factors: current rates (the term before the parentheses), channel gain (the first term inside the parentheses), and interferences to others (the second term inside the parentheses).

If $P_{\max }$ is large enough for every user in the cell to have the minimal distortion, we assign $D_{i}=1$ to everybody. Still, there might be some total transmit power left.

If $P_{\max }$ is not large enough for every user to operate at minimal distortion, the algorithm initially assigns $D_{i}=$ $D_{\max }, \forall i$. If the power is still not enough, it means that there is not enough power to satisfy the group's maximal distortion constraints and the system is in an outage condition. If there is some power left after assigning $D_{i}=D_{\max }, \forall i$, the extra power could be used to reduce distortion. The user that will reduce its distortion is chosen by determining the gradient $\partial P_{\text {sum }} / \partial D_{i}$. If the absolute value of the gradient is small, it means that a reduction in distortion will have a small effect on the total consumed power. For this user, the current rates are low (i.e. the distortion is high), channel gain is good, or interferences to others are small, consequently this user deserves a smaller distortion. In other words, this user can reduce its end-to-end distortion while creating the smallest strain on the available resources. Thus, the algorithm assigns a higher $r_{i}$ to this user to let the distortion become small. Then the gradient is estimated and the rate is assigned again. This process is continued, according to the order of the gradients, until the power is used up. Note that, in practice, there are only a finite number of possible values for $r_{i}$. Therefore, the assignment of a higher $r_{i}$ is done in incremental discrete steps.
TABLE I

Resource Allocation Algorithm

1. Initialization:
If everybody can get $D_{\min }$, then allocate the powers and stop;
else allocate $D_{\max }$ to everybody.
If $P_{\text {sum }}>P_{\max }$, report outage.
2. Repeat:
- Calculate $\left|g_{i}\right|$
- Increase the rate of the user with smallest $\left|g_{i}\right|$ to the next available
discrete rate, unless the rate is $1 / 2$ already.
- If $P_{\text {sum }}>P_{\max }$, return the previous rate allocation and break.
3. Rate and Power Assignment.

On the whole, the adaptive resource allocation algorithm is given in Table I. As we have mentioned before, (6) is extremely difficult to solve by traditional methods in which the complexity grows fast with the number of user $N$ increasing. In the proposed algorithm, the complexity lies in computing the gradients in (13) and calculating the overall transmitted power in (8). So the complexity is $O\left(N^{2}\right)$ and can be easily implemented in practice.

\section{Dynamic System Model}

So far we have considered for the development of the resource allocation algorithm that there is fixed number of users in the system. In reality, the traffic load changes over time as users join or leave the system. Assume that calls enter the cell at a rate $\lambda$ following a Poisson arrival process (i.e. exponential interarrival time) and that the random calls duration follow an exponential distribution with mean $1 / \mu$. The number of admissible users is limited to a maximum $N_{\max }$. Therefore, the stationary probability that there are $n$ ongoing calls in the system is given by:

$$
P[N=n]=\frac{\frac{\rho^{n}}{n !}}{\sum_{i=0}^{N_{\max }} \frac{\rho^{i}}{i !}},
$$

From the study in previous sections, the expected overall distortion with the number of users being fixed, $E(D \mid N=n)$, is known. By using the distribution in (14), the average distortion per call is $E[D]=E[E(D \mid N=n)]=\sum_{n=0}^{N_{\max }} E(D \mid N=$ n) $P[N=n]$.

The maximum admissible number of users $N_{\max }$ can be set based on different design considerations. One straightforward idea would be to limit the number of users based on the processing gain, i.e. $N_{\max }$ has to be less than or equal than the processing gain. In our case we consider the average distortion per call as the performance measure for the call admission policy. Specifically, $N_{\max }$ is selected such that $E(D) \leq d$, where $d$ is a given threshold.

In addition to this, if we consider voice traffic, the load generated by users in a call also changes over time because speech can be considered to follow a two-state (talk spurt and silence) model. In two-way conversation calls, the silent period is roughly $65 \%$ of all the time [17]. Therefore, when the speech is in silence state, there is no need to have high transmission rates or stringent quality constraints, thus reducing interference and potentially increasing the system capacity. In fact, for those users in a silent period, it is possible to assign them the 


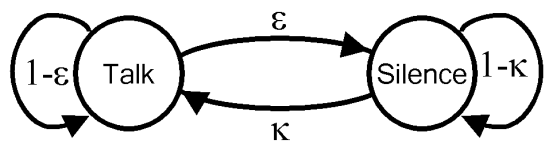

Fig. 2. Two-State Markov Model for Speech Activity

smallest possible resources while still having a good received quality.

\section{Simulation Results}

Since our focus is real time communications, in simulations we used voice as source. We use eighteen speech sequences, both male and female speakers, from the NIST speech corpus [13]. We encoded these sequences using the GSM AMR (Advance Multi-Rate) Narrow-band Speech Encoder [14]. This is a multirate encoder that operates with $20 \mathrm{~ms}$ frames, $5 \mathrm{~ms}$ look-ahead and includes an error concealment mode which was used in simulations. Of the eight possible encoding rates: $12.2,10.2,7.95,7.4,6.7,5.9,5.15$ and $4.75 \mathrm{kbps}$, we used only the six highest ones.

To determine the end-to-end distortion, we choose a perceptually weighted log-spectral distortion measure calculated by numerical approximation of the function

$$
S D(\hat{A}(f), A(f))=\sqrt{\int\left|W_{B}(f)\right|^{2}\left|10 \log \frac{|\hat{A}(f)|^{2}}{|A(f)|^{2}}\right|^{2} d f}
$$

where $A(f)$ and $\hat{A}(f)$ are the FFT-approximated spectra of the original and the reconstructed speech frames, respectively, and $W_{B}(f)$ is the subjective sensitivity weighting function [15]:

$$
W_{B}(f)=\frac{1}{25+75\left(1+1.4(f / 1000)^{2}\right)^{0.69}} .
$$

This distortion is measured on a frame-by-frame basis and then averaged over all frames, including outliers, to further capture the effects of channel errors. We choose this measurement not only because of its good mathematical properties, but also because of its good correspondences to subjective measure [16]. To report the results, we used a normalized distortion measure, which is computed as the ratio of the spectral distortions to that of the speech sequence encoded at the highest rate (12.2kbps) in the absence of channel noise. Note that our assumed distortion-rate performance, as presented in (4), is based on the assumption of a mean squared-error distortion measure. Nevertheless, we noticed through simulations that this assumption remains applicable when using (15) as measure.

Also, for the proposed system, we assumed BPSK modulation. For variable rate channel coder, we choose a memory 4 , puncturing period 8 , mother code rate $1 / 4$ RCPC code in [8] decoded with a soft Viterbi decoder. The total bandwidth was $1.5616 \mathrm{MHz}$. We assumed a channel affected by normalized Rayleigh fading (average power loss equal to 1) and normalized path loss (with propagation constants assumed equal to 1), with a path loss exponent equal to 3 (typical of urban areas). We apply the average orthogoality factor $\alpha$ to all the $\alpha_{k i}$ (to all users). Users are randomly located in a cell with

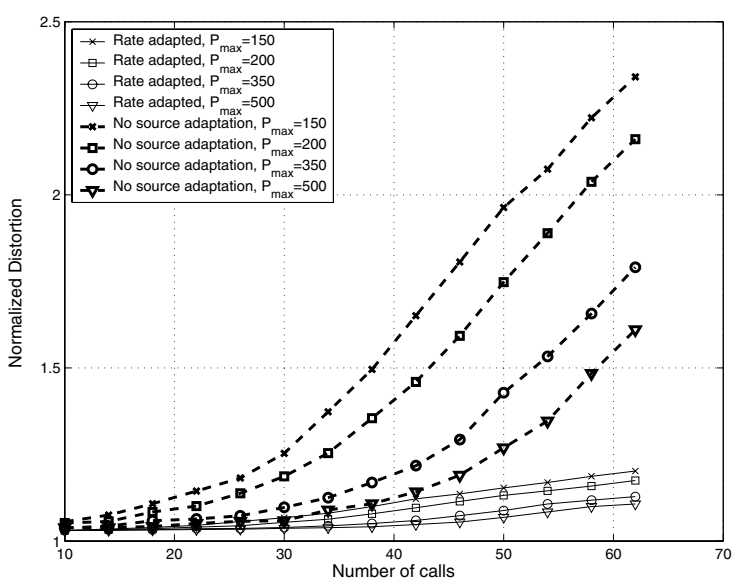

Fig. 3. Normalized Distortion vs. No. of Calls

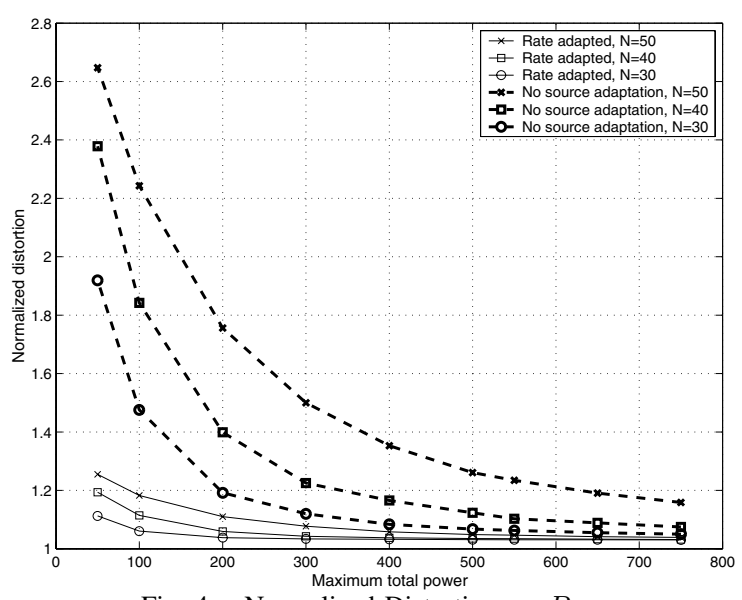

Fig. 4. Normalized Distortion vs. $P_{\max }$

radius $500 \mathrm{~m}$. Background noise level was assumed equal to $10^{-6} . k=3.3 \cdot 10^{-5} \cdot r_{\max }=12.2 \mathrm{kbps}$.

We fix the transmit rate at $24.4 \mathrm{kbps}$ and processing gain at 64 and we analyze the performance of the proposed algorithm as the system becomes increasingly loaded. In Fig. 3, we show the normalized distortion vs. the number of calls with different total transmit powers for the proposed scheme. The figure also includes, for comparison purposes, results for an equivalent CDMA system that shares the same configuration as the proposed scheme but is forced to operate without adaptation. For the case of this equivalent system, all calls operate at a source encoding rate equal to $12.2 \mathrm{Kbps}$ and channel coding rate $1 / 2$. From these results we can draw several conclusions. When the number of users is small, all the schemes with different powers work in the same way. This is because there is enough power to allocate to all users resources so that they operate at minimal distortion. As compared to the equivalent unadapted system with the same outage probability, when the number of users is increased and the system becomes more loaded, the proposed scheme shows a lower normalized distortion. This is because the proposed scheme controls the distortion smoothly by adapting the source and channel coding rates. In particular, if, for example $P_{\max }=350$, we can see that the proposed system can support 30 users with $6 \%$ less distortion, 40 with $12 \%$ and 50 users with $37 \%$ less distortion. 


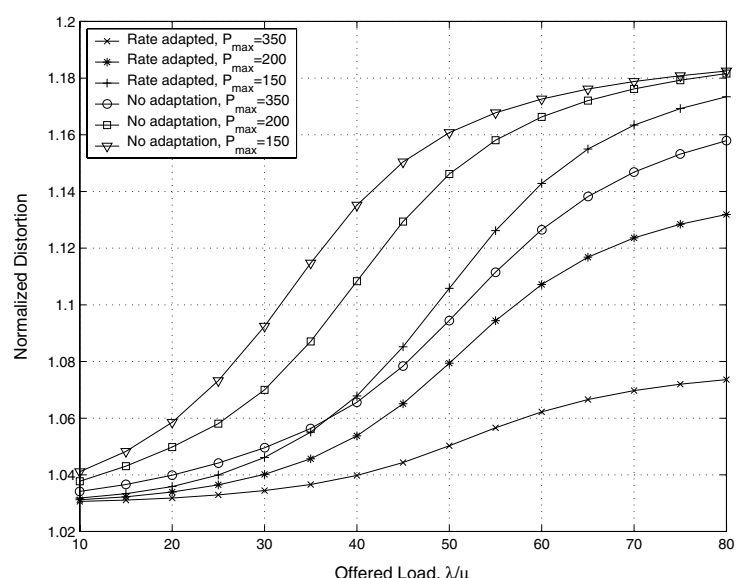

Fig. 5. Dynamic System: Normalized Distortions with $d=1.2$

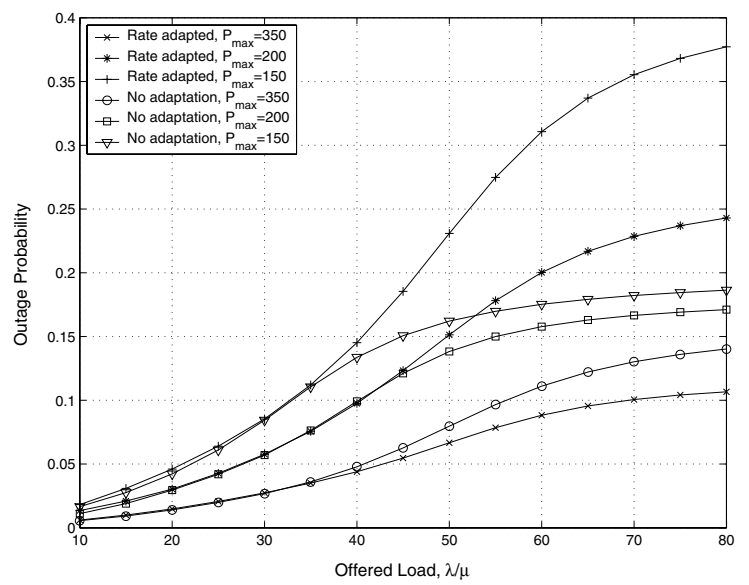

Fig. 6. Dynamic System: Outage Probabilities with $d=1.2$

When the transmitted power is increased, the distortion will be reduced. In Fig. 4, we compared the normalized distortion as a function of the maximal available power for a fixed number of users in the system $(N=30, N=40$ and $N=50)$ that represents different loading conditions on the network. This figure shows that the proposed system is able to deliver the same level of average end-to-end distortion by a much lower maximum total transmit power.

We tested the proposed resource allocation algorithm under dynamic system considerations through Monte Carlo method. In the simulations we fixed the average call duration to 3 minutes, $\mu=1 / 180$, and we measured the performance with different offered loads $\lambda / \mu$. The average talk spurt duration is $1 \mathrm{~s}$. and the average silence duration is $1.35 \mathrm{~s}$. In Figure 5 and Figure 6, we show the average normalized distortions and outage probabilities vs. offered load for a call admission control policy set so that $d=1.2$. We compare the proposed algorithm vs. the fixed algorithm for $P_{\max }=150,200,350$, respectively. We can see that both the normalized distortions and the outage probabilities grow with the offered load increasing, while the proposed algorithm provides much lower distortions and outage probabilities.

\section{CONCLUSiOnS}

In this paper, we design a protocol to smoothly control each user's distortion by varying the source coding rate, channel coding rate and transmitted power in the downlink of a power constrained CDMA system. We develop a fast algorithm to minimize the system overall distortion under the maximal transmitted power and maximal user's distortion constraints, according to different users' current rates, channel conditions, and interferences to others. The algorithm provides an effective solution that in essence allocates target SINRs, from a set of possible values to each user. Compared with a traditional approach, a system that cannot adapt its source and channel coders, the proposed scheme can greatly reduce the overall system distortion and required total transmit power, which, in turn, will increase the maximal number of admissible users. In our study we also consider a dynamic model where the number of calls change over time following a truncated Poisson random process and the speech sources follow a talkspurt-silence model.

\section{REFERENCES}

[1] M. Alasti and N. Farvardin, "SEAMA: a source encoding assisted multiple access protocol for wireless communications", IEEE J. Sel. Areas in Comm., pp. 1682 -1700, Sep. 2000.

[2] A.Sampath, P.S. Kumar, and J.M. Holtzman, "Power control and resource management for a multimedia CDMA wireless system", PIMRC'95.

[3] S. Zhao, Z. Xiong, and X. Wang, "Joint error control and power allocation for video transmission over cdma networks with multiuser detection," IEEE Trans. on Circuits and Systems for Video Technology, vol. 12, no.6, pp.425-437, Jun. 2002.

[4] J. W. Lee, R. R. Mazumdar, and N. B. Shroff, "Downlink power allocation for multi-class CDMA wireless networks", Proceedings of the INFOCOM'2002, N.Y., Jun. 2002.

[5] P. Zhang, S. Jordan, P. Liu, and M. L. Honig, "Power control of voice users using pricing in wireless networks", ITcom'01, 2001.

[6] C.W. Sun and W.S. Wong, "Power control and rate management for wireless multimedia cdma systems", IEEE Trans. on Comm., vol.49, no.7, pp.1215-1226, Jul. 2001.

[7] Z. Han, A. Kwasinski, K. J. Ray Liu and N. Farvardin, "Pizza Party Algorithm for Real Time Distortion Management in Downlink SingleCell CDMA Systems", Proceedings of the 2003 Allerton Conference.

[8] J. Hagenauer, "Rate compatible punctured convolutional (RCPC) codes and their applications", IEEE Transactions on Communications, vol. 36, pp.389-399, April 1988.

[9] F. Adachi, "Effects of orthogonal spreading and rake combining on DSCDMA forward link in mobile radio", IEICE Trans. on Communications, vol.E80-B, no.11, pp.1703-1712, Nov. 1997.

[10] O. Awoniyi, N.B. Mehta, and L.J. Greenstein, "Characterizing the ortogonality factor in WCDMA downlinks", IEEE Trans. on Wireless Communications, vol.2, no.4, pp.621-625, Jul. 2003.

[11] A Gersho and R. M. Gray, Vector quantization and signal compression, Kluwer Academic Publishers, 1992.

[12] N. S. Jayant and P. Noll, Digital coding of waveforms, Prentice-Hall Inc., 1984.

[13] DARPA TIMIT, "Acoustic-phonetic continuous speech corpus CDROM", Document NISTIR 4930, NIST Speech Disk 1-1.1.

[14] ETSI/GSM, "Digital cellular telecommunications system (Phase 2+); Adaptive Multi-Rate (AMR) speech transcoding (GSM 06.90 version 7.2.1 Release 1998)", Document ETSI EN 301704 V7.2.1 (2000-04).

[15] J.S. Collura, A. McCree, and T.E. Tremain, "Perceptually based distortion measurements for spectrum quantization", Proc. 1995 IEEE Workshop on Speech Coding for Telecommunications, 1995.

[16] L. Rabiner and B.H. Juang, Fundamentals of speech recognition, Prentice Hall PTR., 1993.

[17] B.S. Atal, V. Cuperman, and A. Gersho, Speech and audio coding for wireless and network application, Boston: Kluwer Academic, 1993. 